\title{
Robotic Crawler for Inspecting Generators with Very Narrow Air Gaps
}

\section{Conference Paper}

\section{Author(s):}

Fischer, Wolfgang; Caprari, Gilles; Siegwart, Roland; Moser, Roland

Publication date:

2009

Permanent link:

https://doi.org/10.3929/ethz-a-010035891

Rights / license:

In Copyright - Non-Commercial Use Permitted 


\title{
Robotic Crawler for Inspecting Generators with Very Narrow Air Gaps
}

\author{
Wolfgang Fischer, Gilles Caprari, Roland Siegwart, Roland Moser
}

\begin{abstract}
The non-destructive inspection of generator stators is a repetitive and cost-intensive task. For some types with very small entrance gaps (around $9 \mathrm{~mm}$ height), it was even necessary to always remove the rotor.

This paper describes the design and prototype implementation of a new robotic crawler which is only $8 \mathrm{~mm}$ high and thus can inspect all types of generators with the rotor installed. Different solutions of how to realize torque transmission and attraction forces at such a small size are discussed, as well as the design of magnetic guiding wheels with a mechanism to fold them when passing the narrow entrance gap. The paper concludes with some test results - both in an artificial test environment and in real generators - and provides an outlook on future improvements of the prototype.
\end{abstract}

\section{INTRODUCTION}

$\mathrm{T}$ HIS paper describes the design and prototype implementation of a new robotic crawler for the inspection of very thin air gaps in generators. These generators are installed in every power plant and have to be checked regularly for failures, to assure that the power plant works safe and reliable.

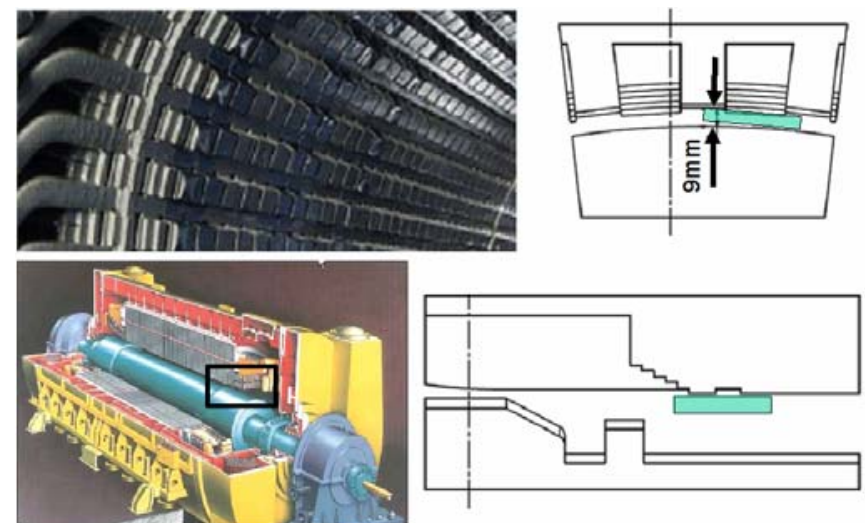

Fig. 1: Sketch of a generator (down, left), with the typical worst-case entrance geometry (right) and the structure of the stator (up, left)

Manuscript received October 15,2008 . Work partially supported by grant KTI 8435.1 EPRP-IW of the Swiss "Bundesamt für Berufsbildung und Technologie" and ALSTOM Power Services, Baden, Switzerland.

Wolfgang Fischer is a PHD student at the Autonomous System Lab at ETH Zürich, Tannenstrasse 3, CLA E18, 8092 Zürich, Switzerland, wfischer@ethz.ch, phone: +41 4463227 40, fax: +41 4463211 81;

Gilles Caprari is a post-doc at the same lab, room CLA E14.1, gilles.caprari@mavt.ethz.ch, phone: +41446322802

Roland Siegwart is the professor of this lab, room CLA E32, rsiegwart@ethz.ch, phone: +41 4463223 58,www.asl.ethz.ch

Roland Moser works as Director R\&D in Inspection Technologies at ALSTOM Power Service, Brown Boveri Str. 7, CH-5401 Baden, roland.moser@power.alstom.com, phone: +41 5620565 09, fax: +41 56 20566 73, www.alstom.ch
As each day of outage can easily cost up to one million euros, the most important goal for these inspections is to be as fast as possible. Thus, ideally the most time-consuming work step - the removal of the rotor - should be avoided by using inspection devices of very small size. These devices should to be small enough to fit between stator and rotor but still mobile enough to move along the stator geometry that is normally formed out of several rows of teeth.

This paper describes a new design that is able to move along these rows of teeth and due to its extremely small height of just $8 \mathrm{~mm}$ can even inspect the smallest generator air gaps without removing the rotor.

The structure is as follows:

Section II provides a brief overlook on existing crawlers for generator inspection, with focus on inspections with the rotor installed. Section III compares different possibilities for providing traction and attraction force, torque transmission and guiding the movement along a straight axial path; to prove that the basic concept with magnetic wheels, spur-gear-transmission and magnetic guiding wheels is very likely one of the best solutions. The first prototype realization is described in Section IV, followed by some test results in Section V. Future improvements in the mechanical design as well as an advanced control strategy and the implementation of a camera-module are discussed in Section VI. In the last section a conclusion is drawn, stressing both on the business relevance of this special application as well as on similar applications where a slightly modified robotic structure could be used.

\section{STATE OF THE ART}

As already shown in the introduction, the simplest way to inspect generators in power plants is to remove the rotor and check the stator manually. However, this work is rather time-consuming and error-prone.

For increasing speed and repeatability, automated devices such as the "Diris Flex" [1] have been developed. Rolling on magnetic tracks, it is able to move along the stator teeth in any inclination. It needs less time to scan a generator than a manual inspector and provides more precise measurement results, but still needs that the rotor is removed for the inspection. 
In order to avoid the removal of the rotor, also some smaller devices have been proposed:

The GE Magic [2] uses three pairs of tracks, with a pneumatic spring in between that allows for spreading between stator and rotor. It is able to move in gaps down to $12.7 \mathrm{~mm}$ ( $=0.5$ inches $)$.

The Siemenst FastGen [3] - similar to the DIRIS Flex uses magnets and tracks and is $22 \mathrm{~mm}$ high.

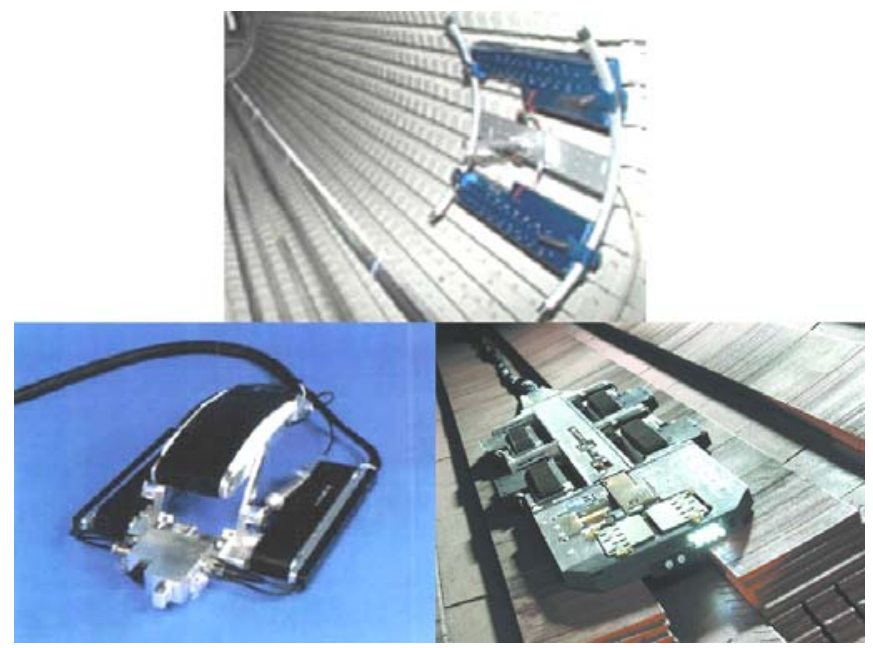

Fig. 2: I made the picture a bit brighter.Current systems for air gap inspection DIRIS Flex[1], GE Magic[2], Siemens FastGen [3]

As none of these devices is able to pass the narrow entrance gaps of the smallest ALSTOM generators with just $9 \mathrm{~mm}$ of height, the goal of this project was to design a crawler at this very small size.

\section{BASIC MECHANICAL CONCEPT}

In order to find the best concept to realize a crawler at this very small height, we analyzed the different alternatives for all the basic mechanical functions - locomotion, torque transmission, adhesion and guiding/keeping a straight path.

\section{1) Locomotion and torque transmission}

As for moving along the stator, the crawler has to overcome the gaps between the teeth, locomotion with only two pairs of wheels is not sufficient. For this reason, most state-of-the-art robots use tracks for locomotion. However, tracks and gearbelts at this very small size are very difficult to produce and not as strong as other transmission elements.

Thus, we decided for several pairs of wheels, with a spur gear transmission in between.

Also other alternatives have been analyzed, but did not seem as promising as the above mentioned.

Worm gears instead of spur gears at similar size would have needed a more complex structure and were calculated to not be as strong enough to transmit the necessary torque.

Using an inchworm-movement with pneumatic actuation similar to the tube crawler built by Tokyo Institute of Technology [4] - was rejected because some inspection sensors need a continuous movement of the crawler.
For generating enough torque, we chose a $6 \mathrm{~mm}$ motor with a 221:1 planetary gearbox from MAXON, as this actuator is one of the strongest that is commercially available at this size - allowing up to $60 \mathrm{mNm}$ of maximum torque.

\section{2) Adhesion}

For keeping in touch to the surface in all possible inclinations from $0^{\circ}$ to $180^{\circ}$, the robot has to adhere itself to the surface. For this function, basically two principles have been used in previous robots: Adhesion with permanent magnets made out of $\mathrm{NdFeB}[1,3]$ or spreading to the rotor using pneumatic springs [2]. In order to have a low system complexity with few parts, we decided for magnets. Other adhesion principles such as gecko-hairs or pneumatic vacuum suction have not been analyzed in too much detail, as these principles are not strong enough at this very small size, more difficult to combine with wheeled vehicles and/or not yet mature enough for industrial applications with high needs for reliability.

To place permanent magnets on a wheeled vehicle, there basically exist two possibilities: In the structure [5] or in the wheels [6]. Vehicles with magnets in the structure have fewer problems due to attracting ferromagnetic dirt particles that then stick on the wheels and reduce the attraction force and the friction coefficient towards the surface. They also allow for using standard wheels with rubber tires that are optimized for very high friction coefficients. However, placing the magnets in the structure needs extra space there and thus increases the robot size. Furthermore, if some ground clearance is needed to prevent the structure magnets touching when passing surface irregularities, they have to be placed rather far away from the surface - resulting in a significantly reduced magnetic force.

In our case, the ground clearance should be around $1 \mathrm{~mm}$ due to small paint drops on the surface. With this value, we compared both options - structure magnets vs. magnetic wheels.

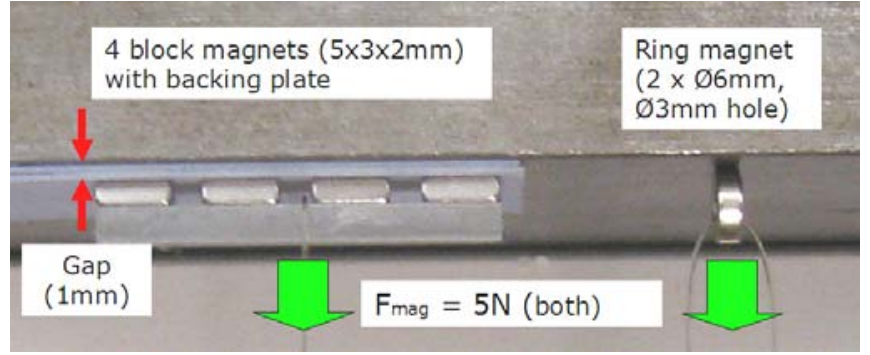

Fig. 3: Comparison of a magnet assembly in the structure (gap 1mm) and a magnetic wheel

For the structure magnet assembly, we chose the arrangement proposed by Shang et al [5], which uses a steel plate at the back of the magnets (backing plate) to achieve high forces even if there is a notable gap between magnets and surface. With an array of 4 block magnets $(\mathrm{NdFeB}$, $5 \times 3 \times 2 \mathrm{~mm}$ ), a $3 \times 3 \mathrm{~mm}$ backing plate and a $1 \mathrm{~mm}$ gap to the surface we measured $5 \mathrm{~N}$ of maximum holding force. The 
same value can also be achieved with a single ring magnet that directly rolls on the surface - using far less space and mass. Or in other words: Significantly higher forces can be achieved, when several of these ring magnets are placed. For this reason, we decided to use magnetic wheels instead of magnets in the structure.

To further increase the adhesion force with these very small magnetic wheels, we used the wheel design proposed by Guy [7] - with rims on both sides of a ring magnet that conducts the force better into the ground and thus increases the magnetic force around 3 times. To achieve even higher forces, we did not only put one wheel on each shaft, but 4 double wheels, similar as we had already done it for another robot that was designed for very thin and fragile surfaces [6].

During its operation, the robot only has to drive horizontally and never needs to climb vertical walls. However, it has to carry rather high payloads when driving upside down. Thus, the goal to realize high adhesion forces has been seen more important than improving the friction coefficient to the surface. For this reason, the magnetic wheels are not covered with rubber. By not using a rubber tire, also the manufacturing process could be done much simpler and cheaper.

With this design, we could achieve an adhesion force of $50 \mathrm{~N}$ for each shaft - which is around 10 times the force of one ring magnet alone or 2.5 times the force of 4 ring magnets without rims.

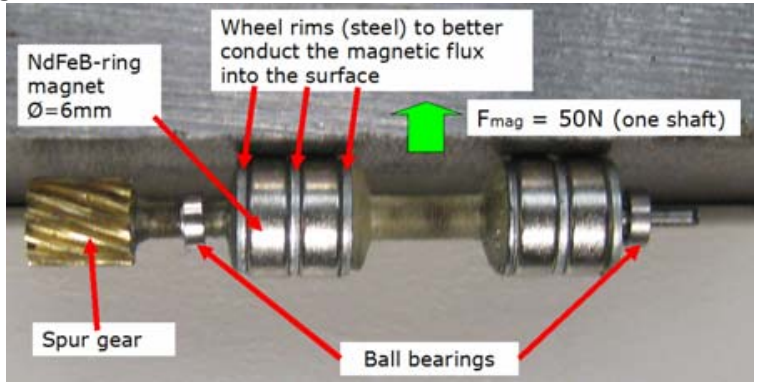

Fig. 4: Shaft with 2 double magnetic wheels, hanging on a block of steel

3) Guiding

For guiding the vehicle along a straight path, previous robots basically use two possibilities: 2 units with skidsteering and a line-following-algorithm (GE Magic [2]) or mechanically guiding on the side of 2 rows of teeth (DIRIS Flex [1], Siemens FastGen [2]). Both alternatives have disadvantages: The solution with two units and skid steering results in an increased system and control complexity; and the mechanical guiding elements have to be adapted for each different generator to inspect, as the distance between the teeth rows is not the same.

Thus, we implemented a guiding that only needs one row of teeth. To do so, our guiding wheels are magnetic - similar to the main wheels. Like this, they can also transmit negative normal forces (= pulling, not only pushing). One row is then enough and an adaptation to different teeth distances is not necessary any more. This method solves in an elegant way the guiding of the robot on its linear path.

\section{4) Folding of guiding elements}

In some generators, the entrance is very narrow so that there is no space for entering the guiding wheels in a standard way. For also entering these generators, the structure that holds the lateral guiding wheels can be folded flat when passing the entrance gap. For actuating this DOF, we again chose a $6 \mathrm{~mm}$ DC motor from MAXON, but this time with the highest available reduction factor in the gearbox (854:1). With this gearbox, we could assure that the transmission is quasi self-blocking and thus does not need power to keep its position; and also has enough torque to detach the guiding wheels when the robot is removed from the generator. The sequence how the robot is inserted and afterwards removed is as follows: At first, the guiding rolls are folded flat (see small sub-image in Fig. 5) to pass the narrow entrance geometry (see Fig. 1). Afterwards, the guiding rolls are clapped out (see main image in Fig. 5) to provide the lateral guiding. Before removing the robot, the guiding rolls are folded flat again.

\section{PROTOTYPE REALIZATION}

According to this concept, we realized a first prototype, as it can be seen in Fig. 5. Its basic drive train consists of the 10 shafts with magnetic wheels, spur gears and ball bearings that are glued together as one part, intermediate axes and the actuator (DC-motor with 221:1 gearbox). The structure is made out of stereolithographic plastic. On one side, there is the holder for the magnetic guiding rolls, which can be clapped in and out with a second actuator.

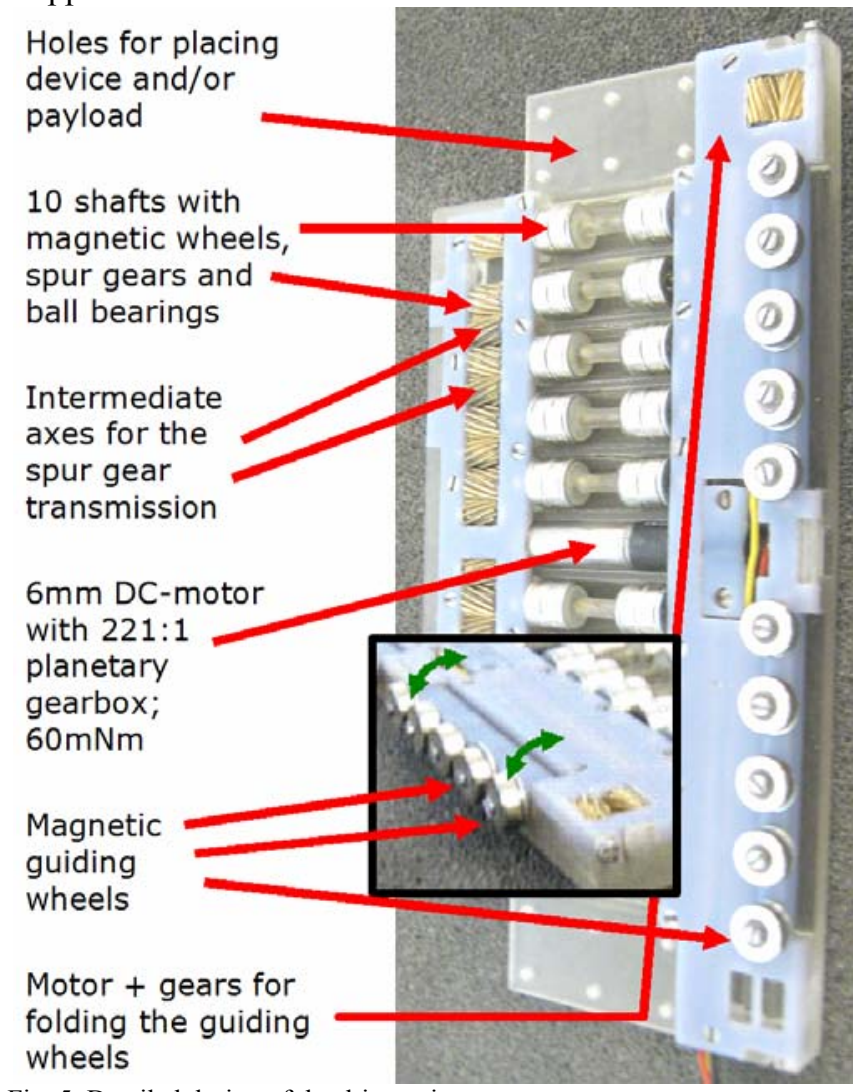

Fig. 5: Detailed design of the drive unit 


\section{TEST RESUlts}

We tested the crawler in several configurations to better estimate the effects of each parameter that can be changed and thus be optimized easily. These tests were performed on a test environment formed out of 3 steel blocks that have been attached to a L-profile - with gaps of variable lengths in between and the two worst-case-positions: hanging on the side $\left(90^{\circ}\right)$ and hanging upside down $\left(180^{\circ}\right)$. To estimate if the robot will be able to carry the mass of a camera, some extra sensors, electronics and cables, we sometimes put some payload on the crawler.

\section{A. Tests with a reduced number of wheels}

At first, we tested with a reduced configuration, with 4 powered wheel units and two non-powered ones:

In total, the prototype could move in all possible inclinations and was able to carry $140 \mathrm{~g}$, which is equivalent to 2.8 times its own mass $(50 \mathrm{~g})$. We measured a speed of around $1.8 \mathrm{~m} / \mathrm{min}$. Also passing gaps of up to $10 \mathrm{~mm}$ was not a problem.

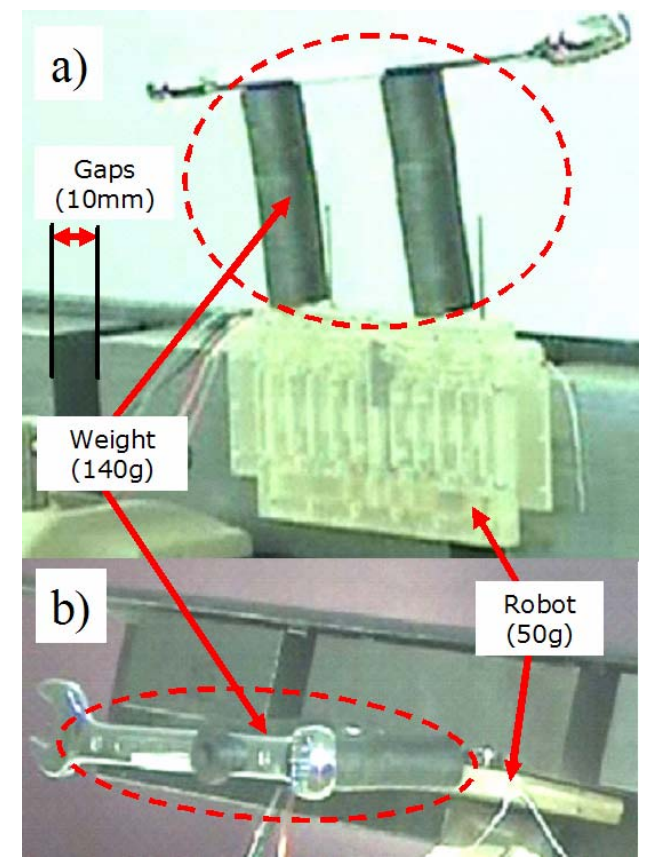

Fig. 6: Tests with only 4 powered and 2 non-powered wheels and a payload of $140 \mathrm{~g}$ ( $=2.8$ times the robot's own mass), in $90^{\circ}$ (a) and $180^{\circ}$ (b) position

With these results, we could be sure that the full configuration would easily be able to move with enough safety margin and be able to carry a camera and some sort of inspection sensor.

\section{B. Tests with the full number of wheels}

The full configuration was then built and tested, both in the test environment and in the real generator. With this configuration, it was possible to even carry up to $500 \mathrm{~g}$ and to pass even bigger gaps between the teeth. Up to $30 \mathrm{~mm}$ were tested successfully - corresponding to the maximum gap size that can be found in ALSTOM generators.
Due to the higher internal friction losses with 10 instead of only 4 powered wheels, the speed at nominal voltage (6V) was slightly slower: $1.2 \mathrm{~m} / \mathrm{min}$ instead of $1.8 \mathrm{~m} / \mathrm{min}$. This value still seemed to be enough. The power consumption was approximately the same in both configurations $-0.5 \mathrm{~W}$.

As the very first centimeters of the entrance are formed by a surface geometry that cannot be passed with the vehicle (see Fig. 1), we put an aluminum bar on one side in order to place the robot just after that short difficult zone. For equilibrating the mass, we also put the same aluminum bar on the other side. In the final version, one of these aluminum bars will then be substituted by the final payload.

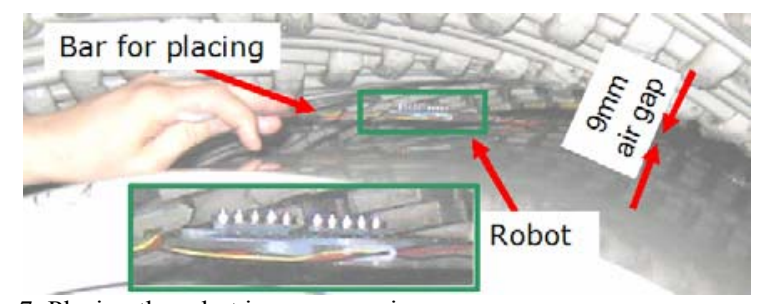

Fig. 7: Placing the robot in a narrow air gap

The tests were performed in two different generator stators: One with small diameter $(\varnothing \approx 1.5 \mathrm{~m})$ and unpainted, one with larger diameter $(\varnothing \approx 3 \mathrm{~m}$ and painted $)$.

In the unpainted stator, the prototype performed well in all inclinations. In the painted one, on some paths it had problems to pass some of the biggest paint drops - with heights up to $2 \mathrm{~mm}$.

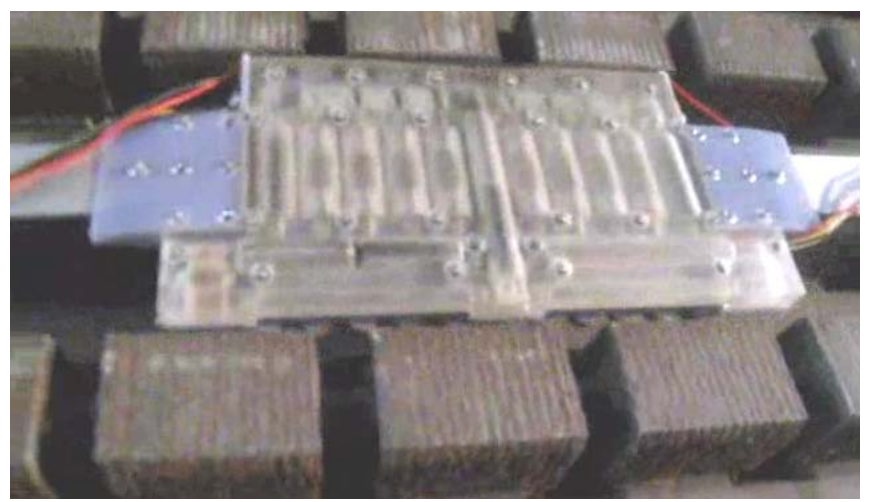

Fig. 8: Robot in an unpainted stator of a generator, where it moved without problems

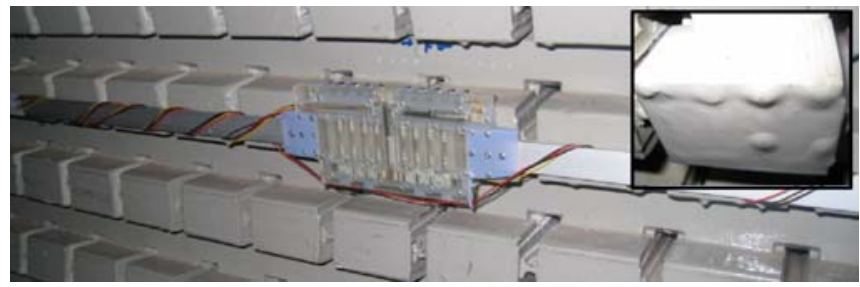

Fig. 9: Robot in a painted stator of a generator, where it sometimes had problems due to big paint drops 
This problem could be addressed in further versions, where some sort of suspension will be integrated to better adapt the very small wheels to the rather big paint drops. However, as most generators do not have these paint drops, solving this problem was not seen as first priority.

\section{CONCLUSION AND OUTLOOK}

A mechanical prototype for a new type of miniature air gap crawler for generator inspection has been realized, at a height that had not been reached before. To achieve this extremely small height of only $8 \mathrm{~mm}$, we used an intelligent way of torque transmission and magnetic guiding wheels that can be folded flat when the crawler passes the entrance gap. The prototype performed well - both in the test environment and in real generators.

The new crawler will be able to enter all types of generators that can be found in power plants without removing the rotor - even the ones with the most narrow entrance gaps. This might have a major economical impact in plant inspection.

Future work will focus on adapting the structure parts for manufacturing it out of more stable material (milled out of aluminum instead of 3D-printed plastics); placing an encoder and a sensor for counting the teeth in order to localize the robot's position at any time and do speed control; and an interface for camera and NDT sensors.

\section{REFERENCES}

[1] DIRIS Flex:

R. Moser, B. Mark; “Automated Robotic Inspection of Large Genertor Stators"; Int. Conference of Advanced Intelligent Mechatronics (AIM); 2007

and http://www.inspectionrobotics.com/projects.htm

[2] GE Magic:

D.T. Roney, R. J. Zawoyski; “Generator In-Situ Inspections”; GE Power Systems, found on http://www.gepower.com/prod_serv/serv_for/generators/en/testing_in sp/ndt.htm

[3] Siemes FastGen:

D. Schoeler, P.O. Miranda; "Improved Unit Reliability \& Availability Through Optimised Predictive Maintenance".; Siemens AG, Power Generation; 2003

[4] Tube crawler with pneumatic inchworm movement: Y. Kondoh, S. Yokota; "Micro in-pipe mobile machines by making use of an electro-rheological fluid", IEEE/RSJ International

Conference on Intelligent Robots and Systems,Vol.3,pp.1672-7; 1997

[5] Climbing robot with magnetic arrays in the structure:

J. Shang, B. Bridge, T. Sattar, S. Mondal, A. Brenner; "Development of a climbing robot for inspection of long weld lines"; Int. Journal of Industrial Robots; Vol. 35, Issue 3, pp. 217-223; 2008

[6] Several magnetic wheels in parallel, for high forces at small size: W. Fischer, F. Tache, R. Siegwart; "Inspection System for Very Thin and Fragile Surfaces, Based on a Pair of Wall Climbing Robots with Magnetic Wheels", Int. Conference on Intelligent Robots and Systems (IROS); 2007

[7] First description of a magnetic wheel:

W. Guy, "Magnetic Wheel”, US Patent 3,690,393, September 1972 